\title{
Los abuelos favoritos desde la percepción de preadolescentes de la ciudad de Armenia
}

\section{The favorite grandparents from the perception of preadolescents in Armenia city}

\author{
Ruiz Soto María Claudia*; Pineda Chacón Olga Mercedes*; Valencia Restrepo Sandra Milena*; \\ Grupo de Investigación en Desarrollo
}

Recibido: mayo 7 de 2009

Aceptado: octubre 1 de 2009

Correspondencia: Programa de Gerontologia, Universidad del Quindio, Avenida Bolivar calle 12 norte Armenia Quindio, Email: claruso2@yahoo.es

\section{RESUMEN}

Esta investigación forma parte de los estudios sobre Abuelidad, que adelanta el Grupo de Investigación sobre Desarrollo de la Universidad del Quindío. Se describe, desde la percepción de preadolescentes de la ciudad de Armenia, la figura del abuelo favorito a partir de características del abuelo (edad, sexo, familia de origen, ocupación, ubicación geográfica y frecuencia de contacto) y como ésta varía en función de las características del nieto.

La muestra estuvo conformada por 600 preadolescentes de distintas instituciones educativas a los cuales se les aplicó un instrumento sobre la relación abuelo-nieto. Los datos fueron procesados en Stadistical Product and Service Solutions (SPSS), se utilizó estadística descriptiva, en la identificación de la figura del abuelo favorito y para establecer diferencias significativas en función las características del nieto, el Chi-cuadrado y la prueba U de Mann Whitney.

Los resultados indican que los nietos eligen como abuelo favorito en mayor porcentaje a abuelas (81.4\%), de línea materna (62.1\%), entre 60 y 70 años (43.8\%), jubilados o amas de casa (72.8\%), viven en la misma ciudad que sus nietos (32.7\%), les ven casi todos los días (47.4\%). Se encontró que las características de la figura del abuelo presenta variaciones en función de características del preadolescente. Así, la edad del abuelo favorito varía de acuerdo con la estructura familiar y el orden de nacimiento del nieto, cuando la madre trabaja, los nietos prefieren en mayor proporción a las abuelas maternas; la distancia geográfica y la frecuencia de contacto entre abuelos favoritos y nietos varían en función de la estructura familiar, el número de hermanos y el orden de nacimiento del preadolescente

Palabras clave: Relaciones intergeneracionales, percepción del abuelo favorito.

\section{ABSTRACT}

This research forms a part of the studies on Grandparenthood, developed by the Group of Research on Development from the University of Quindío. The figure of the favorite grandparent is described, from the perception of preadolescents in the city of Armenia, from characteristics of the grandparent (age, sex, family of origin, occupation, geographical location and frequency of contact) and how it changes depending on the characteristics of the grandchild. The sample was comformed by 600 preadolescents from different educational institutions, to whom we applied an instrument on the relation grandparentgrandchild. The data was processed in Stadistical Product and Service Solutions (SPSS). Descriptive statistics was used in the identification of the favorite grandparent, and for establishing significant differences in function of the characteristics of the grandchild, the Chi-square and the test U of Mann Whitney.

The results indicate that the grandchildren chose their grandmothers as their favorite grandparent (81.4\%), on the mother family (62.1\%), between 60 and 70 years old (43.8\%), who are pensioners or housewives (72.8\%), live in the same city that her grandsons (32.7\%), and see them almost every day (47.4\%).It was found that the characteristics of the figure of the grandparent present variations depending on characteristics of the preadolescent. This way, the age of the favorite grandparent changes in agreement with the familiar structure and the order of birth of the grandchild, when the mother works, the grandsons prefer in major proportion the mother grandmother; the geographical distance and the frequency of contact between favorite grandparents and grandsons change depending on the familiar structure, the number of brothers and the order of birth of the preadolescent.

Key words: intergenerational Relations, perception of the favorite grandfather.

\footnotetext{
*Universidad del Quindío. Programa de Gerontologìa Facultad de Ciencias Humanas
} 


\section{INTRODUCCIÓN}

no de los aspectos que caracteriza al siglo XX es, sin duda, los cambios en todos los niveles de la estructura social (económico, político, religioso, científico, tecnológico, familiar, educativo, entre otros), cambios propios de la postindustrialización. En el umbral del siglo XXI, estos siguen su curso, algunos a pasos agigantados, influenciándose mutuamente y modificando de manera notable a nivel estructural y organizacional las diferentes instituciones sociales. Tal es el caso del sistema familiar en Colombia.

Los diferentes cambios ocurridos en la institución familiar a nivel estructural y funcional, son producto de la influencia e interacción de cada uno de sus miembros en un ambiente de continua evolución. Todo cuanto acontece en las diversas esferas de la estructura social, afecta en mayor o menor medida a la familia como grupo social, dependiendo del grado en que esta se involucre en cada una de ellas y de las relaciones que se mantienen entre sus miembros.

De manera particular, fenómenos como el aumento de la esperanza de vida y la población mayor de 60 años, la crisis económica, vinculación de la mujer al mercado laboral, migración externa, madresolterismo, divorcios, embarazos en adolescentes, entre otros, hacen que la composición de la familia y las funciones de sus miembros se modifiquen y por consecuencia las relaciones familiares (Viguer, 1996; Hacia una política de vejez y envejecimiento: Quindío un departamento para todas las edades, 2009; Huérfanos de Padres Vivos, Procuraduría General de la Nación; Plan de Salud Territorial, periodo 2008-2011). En este sentido, en la familia actual coexisten varias generaciones, en las que cobran especial importancia las relaciones entre abuelos y nietos. Para los abuelos participar en las familias hace que su función social sea mas significativa, al punto que las relaciones intergeneracionales se convierten en un componente de calidad de vida, precedida solo por el estado de salud, las habilidades funcionales y la situación económica de la familia en general y del adulto mayor en particular (Fernández Ballesteros, 2001 cit. En Castañeda y cols., 2004; Osuna, 2006).

Para el adulto mayor, ser abuelo significa desempeñar este rol como uno de los muchos que se realizan a lo largo del ciclo vital, adaptándose a los diversos cambios acaecidos en la estructura social, sobre todo en la esfera familiar. Convertirse en abuelo es un suceso que ocurre en un momento determinado de la vida y que viene dado no por exigencias internas, sino por factores exógenos, es decir, el rol de abuelo es impuesto. El desempeño de este nuevo rol, ya sea de manera positiva o menos satisfactoria, esta condicionado por por múltiples aspectos como la edad y el género de los abuelos y nietos, la línea familiar, la ubicación geográfica, la estructura familiar, el orden de nacimiento del nieto, la duración del mismo rol y el alternar la realización de sus actividades cotidianas con el ser abuelo, (Kennedy, 1992; Strom y Strom, 1997; Smith, 1995; Roberto y Stroes, 1992; Van Ranst, Verschueren y Marcoen, 1995; Creasey y Kaliher, 1994; Kennedy, 1992; Pinazo, 1999; Emick y Hayslip, 1996; Bengtson, 1995; Mckay y Caverly, 1995; Szinovacz, 1998; Castañeda y cols., 2004; Celdrán, 2004; Osuna, 2006). Estas características influyen en la elección que los preadolescentes hacen del abuelo favorito, quien se constituye así en el abuelo mas cercano y significativo para su desarrollo emocional, cognitivo y moral.

En este sentido, la relación permanente de los abuelos con los nietos, convierte a los mayores en agentes importantes de socialización. A través del diálogo y las actividades que comparten, el abuelo al igual que los progenitores le brinda a los jóvenes, las pautas cognitivas y afectivas, los valores, los conocimientos y las habilidades que le permitirán actuar y participar eficazmente en la sociedad (Rico, Serra, Viguer 2001; López y Cantero, 1999; Kolhberg, 1999; Pineda y Ruiz, 1996; Rodrigo y Palacios, 1998). Los niños que mantienen altos niveles de interacción con agentes positivos de socialización, reciben estimulación y presentan mejores niveles de desarrollo cognitivo, afectivo y social (ClarkStewart, 1978; Parke y Tinsley, 1981; Power y Parke, 1982; Wachs y Gruen, 1982, cit. en Ochaíta y Espinosa,1995; Pinazo, 1999). De ahí que, el desarrollo social, cognitivo, moral y afectivo del niño, y posteriormente del adulto, se verá afectado por el estilo de socialización que el abuelo y otros agentes de socialización utilicen (Baumrind, 1971. Cit. en Musitu y Cava 2001).

\section{METODOLOGÍA}

La población de preadolescentes, a partir de datos provenientes de la Secretaría Municipal de Educación.de Armenia, se describe en la siguiente tabla:

Tabla 1 . Descripción del universo

\begin{tabular}{|c|c|c|c|}
\hline \multicolumn{2}{|c|}{$\begin{array}{c}\text { No de alumnos por grado } \\
\text { de escolaridad }\end{array}$} & \multicolumn{2}{|c|}{ Tipo de Institución } \\
\hline \multirow{2}{*}{5} & \multirow{3}{*}{5.091 niños (as) } & Públicos & 247 \\
\cline { 3 - 4 } & & Privados & 33 \\
\cline { 3 - 4 } & & Convenio & 10 \\
\hline \multirow{2}{*}{6} & \multirow{3}{*}{5.432 niños (as) } & Públicos & 271 \\
\cline { 3 - 4 } & & Privados & 31 \\
\cline { 3 - 4 } & & de Convenio & 8 \\
\hline
\end{tabular}


La muestra fue seleccionada mediante muestreo aleatorio estratificado, y estuvo conformada por 600 preadolescentes, niños $(50,2 \%)$ y niñas $(49,8 \%)$, de 10 años $(45 . / \%), 11$ años (40.6\%), y 12 años (13.7\%), estudiantes de 5o grado (48.3\%) y 6ㅇ (51.7\%). La representatividad del tipo de institución educativa es: colegios públicos $86.3 \%$; colegios de convenio $3 \%$; y colegios privados $10.7 \%$.

\section{Instrumentos.}

Se adaptó un cuestionario con base en el utilizado por Rico, Serra y Viguer (2001). El cual recopila datos de identificación del niño, datos de identificación familiar y datos de identificación de la figura de abuelo favorito. Las Variables que se analizaron en este trabajo se presentan en el siguiente tabla:

Tabla 2. Variables

\begin{tabular}{|c|c|}
\hline \multicolumn{2}{|c|}{ Características del Abuelo Favorito } \\
\hline \multirow{3}{*}{ Edad } & Menores de 60 años \\
\hline & De 60 años a 70 años \\
\hline & Mayores de 70 años. \\
\hline \multirow{2}{*}{ Género } & Hombres \\
\hline & Mujeres \\
\hline \multirow{4}{*}{$\begin{array}{l}\text { Familia } \\
\text { de } \\
\text { Origen }\end{array}$} & Abuela materna \\
\hline & Abuela paterna \\
\hline & Abuelo materno \\
\hline & Abuelo paterno \\
\hline \multirow{2}{*}{ Ocupación } & Trabaja \\
\hline & No trabaja \\
\hline \multirow{5}{*}{$\begin{array}{l}\text { Distancia } \\
\text { Geográfica }\end{array}$} & En la misma casa \\
\hline & En el mismo barrio \\
\hline & En la misma ciudad \\
\hline & En el mismo departamento \\
\hline & Otro departamento. \\
\hline \multirow{5}{*}{$\begin{array}{l}\text { Frecuencia } \\
\text { de } \\
\text { Contacto }\end{array}$} & Nunca \\
\hline & En vacaciones únicamente \\
\hline & Menos de una vez al mes \\
\hline & Fines de semana \\
\hline & Casi todos los días. \\
\hline \multicolumn{2}{|c|}{ Características del Nieto } \\
\hline \multirow{3}{*}{ Edad } & 10 años \\
\hline & 11 años \\
\hline & 12 años \\
\hline \multirow{2}{*}{ Género } & Hombres \\
\hline & Mujeres \\
\hline
\end{tabular}

\begin{tabular}{|c|l|}
\hline \multirow{4}{*}{$\begin{array}{c}\text { Número } \\
\text { de } \\
\text { hermanos }\end{array}$} & Hijo único \\
\cline { 2 - 2 } & Un hermano \\
\cline { 2 - 2 } $\begin{array}{c}\text { Orden } \\
\text { de } \\
\text { nacimiento }\end{array}$ & Dos o más hermanos \\
\cline { 2 - 2 } & $2^{\circ}$ \\
\cline { 2 - 2 } & $3^{\circ}$ o más \\
\hline \multirow{2}{*}{$\begin{array}{c}\text { Estructura } \\
\text { familiar }\end{array}$} & Biparental \\
\cline { 2 - 2 } & Monoparental \\
\cline { 2 - 2 } & Con otra persona \\
\hline \multirow{2}{*}{$\begin{array}{c}\text { Ocupación } \\
\text { madre }\end{array}$} & Labores remuneradas \\
\cline { 2 - 2 } & Labores no remuneradas. \\
\hline
\end{tabular}

Procedimiento.

Durante la adaptación del cuestionario, se realizaron pruebas piloto para lograr que los niños de 10 a 12 años entendieran las preguntas y las opciones de respuesta al tiempo que permitiera aumentar la fiabilidad y disminuir las posibilidades de sesgo. Se validó por criterio de jueces.

Una vez terminado el instrumento se procedió a acceder a las instituciones educativas, donde se contó con el apoyo de la Secretaría Municipal de Educación en Armenia, quienes se encargaron de enviar oficios a los rectores autorizando la aplicación del cuestionario a estudiantes de 5y 60 .

La información se recolectó por cursos, previa explicación del investigador, quien dirigía el proceso, con una duración de 45 minutos en promedio por grupo.

\section{Procesamiento de la información.}

Los datos fueron procesados en Stadistical Product and Service Solutions (SPSS). En las investigaciones se utilizó tanto estadística descriptiva para describir las características de la figura del abuelo favorito, como inferencial para establecer diferencias, de la figura de abuelo favorito en función de las características del nieto. La prueba de significación utilizada fue el Chi-cuadrado, prueba no paramétrica apropiada para las variables de este trabajo, que no presentan una distribución normal. En aquellos casos en que se obtuvieron frecuencias esperadas inferiores a 5 en más de una casilla al aplicar el chi-cuadrado, se procedió a utilizar la prueba $U$ de Mann Whitney para tener mayor fiabilidad en el manejo de los datos.

\section{RESULTADOS Y DISCUSIÓN}

Figura de abuelo favorito.

En este apartado se exponen, los resultados referentes a las características de los abuelos favoritos reportadas por sus nietos preadolescentes. 
Edad.

Esta es una variable que condiciona la percepción del adulto como abuelo favorito y en consecuencia la forma como este desempeñe el rol. Al indagar a los preadolescentes de Armenia sobre sus preferencias respecto a sus abuelos, se encuentra, que los nietos eligen con mayor frecuencia a los abuelos con un rango de edad entre 60 y 70 años, seguido por los menores de 60 años y en menor proporción por los mayores de 70 años.

Un alto porcentaje de los abuelos favoritos, entre los 60 y 70 años están jubilados o son amas de casa, por esto disponen de más tiempo para dedicar a sus nietos y aún poseen habilidades y capacidades físicas para disfrutar con los nietos, ya sea que participen de manera continua o esporádica en su cuidado.

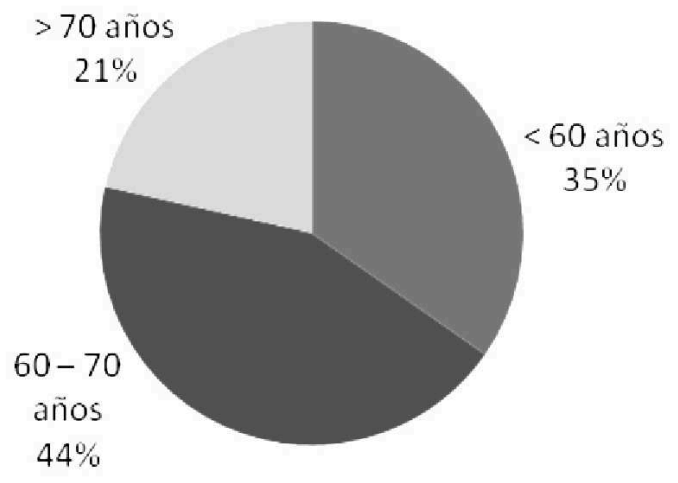

Gráfico 1. Porcentajes de edad de los abuelos favoritos.

En general estos datos apuntan en la misma dirección de diversos estudios realizados por autores como Kahana y Kahana (1970), Creasey y Kaliher (1994), Smith (1995), Van Ranst, Verschueren y Marcoen (1995), Emick y Hayslip (1996), Strom y Strom (1997), y Pinazo (1999), para quienes la edad del abuelo influye en la relación y en la percepción que los nietos tienen de esta.

\section{Género.}

En diferentes estudios sobre las relaciones intergeneracionales, el género es otra de las variables consideradas, hallándose que los nietos perciben de manera más favorable la relación con las abuelas. Estas son valoradas por los menores como personas que influyen en sus vidas, sienten mayor intimidad con ellas que con sus abuelos a la vez que realizan más actividades con estas (Neugarten y Weinstein, 1964; Cherlin y Furstenberg, 1985; Belsky, 1996; cit. en Rico, Serra y Viguer, 2001). Lo que coincide con la elección del abuelo favorito de los niños y niñas de Armenia, quienes han elegido en mayor proporción a las abuelas con una marcada diferencia respecto a los abuelos.

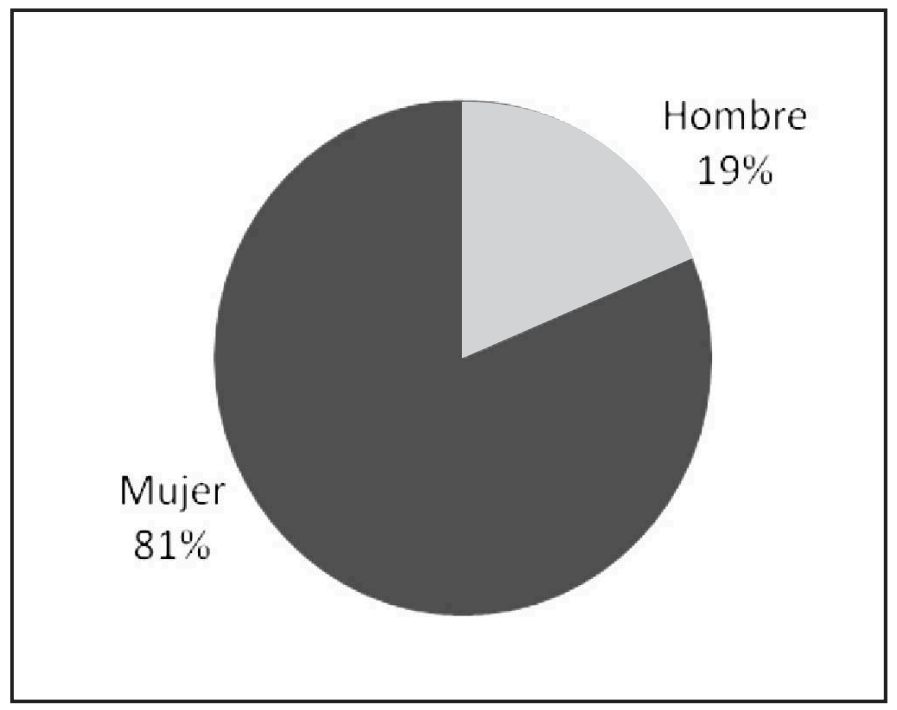

Gráfico 2. Porcentajes de abuelos favoritos según el género-

Se observa la tendencia de la mujer quindiana a seguir teniendo una estrecha relación materna que reflejan y transmiten a sus propias hijas y nietos; si la madre está en continua comunicación con la abuela dándose apoyo y cariño, el niño también es partícipe de esta interacción, por lo cual es probable que desarrolle mayor empatía con la abuela materna.

Por tradición, hombres y mujeres son socializados de acuerdo a las conductas, actitudes e intereses que se consideran apropiados, según el género, dentro de su cultura, lo cual difiere por cuanto aquello que puede ser normal para un hombre en cierta sociedad, puede ser inadecuado en otra y así mismo ocurre con las mujeres (Spera y Matto, 2007). Bajo los parámetros que fueron educados los abuelos quindianos, se espera que las mujeres se dediquen a todas aquellas actividades concernientes a las labores del hogar y el cuidado de los pequeños, mientras los hombres se asocian con tareas más rudas y que realizan por lo regular fuera de casa. Estos roles se asumen desde edades tempranas, sobre todo a través del juego (Ruiz, Pineda y Valencia, 2006), pues mientras al niño se le obsequian carros, balones y se le prohíbe actuar de manera delicada, las niñas reciben baterías de cocina y muñecas, al tiempo que se les exige delicadeza y actitud de servilismo hacia el hombre. Los resultados de este trabajo concuerdan con el estudio de Roberto y Stroes (1992), quienes resaltan que las abuelas hacen un aporte importante al participar en la educación de los nietos, anotando que ellas influyen de manera significativa en el sistema general de creencias de sus nietos y nietas. Así mismo, para Kennedy (1992), el género del abuelo influye en la calidad de la relación, resaltando la cercanía de los nietos hacia las abuelas y sintiéndose más comprendidos por estas que por los abuelos del género masculino. 
En otras investigaciones, coincidiendo con este estudio, se establece que el género del abuelo marca el desempeño del rol y su percepción. Según Kennedy (1992), McKay y Caverly (1995), y Block (2000), las abuelas están más próximas a sus nietos compartiendo más actividades, intimidad y afecto, mientras los abuelos son más distantes a nivel emocional.

\section{Familia de origen.}

Son varías las razones por las cuales la relación más íntima se da entre los nietos y las abuelas de la línea materna, por una parte, puede influir que los abuelos maternos por lo general son más jóvenes que los paternos. Por otra parte, las mujeres mantienen mayor frecuencia de contacto con sus progenitores, siendo el lazo madre-hija, más fuerte que el lazo madre-hijo (Smith, 1991).

Los resultados señalan que, de las abuelas, los nietos prefieren a la abuela de la línea materna (60.4\%) mientras que la abuela paterna es elegida en segunda instancia (21.1\%). En menor proporción está el abuelo de la línea materna (11.7\%) y en último lugar el abuelo paterno (6.8\%).

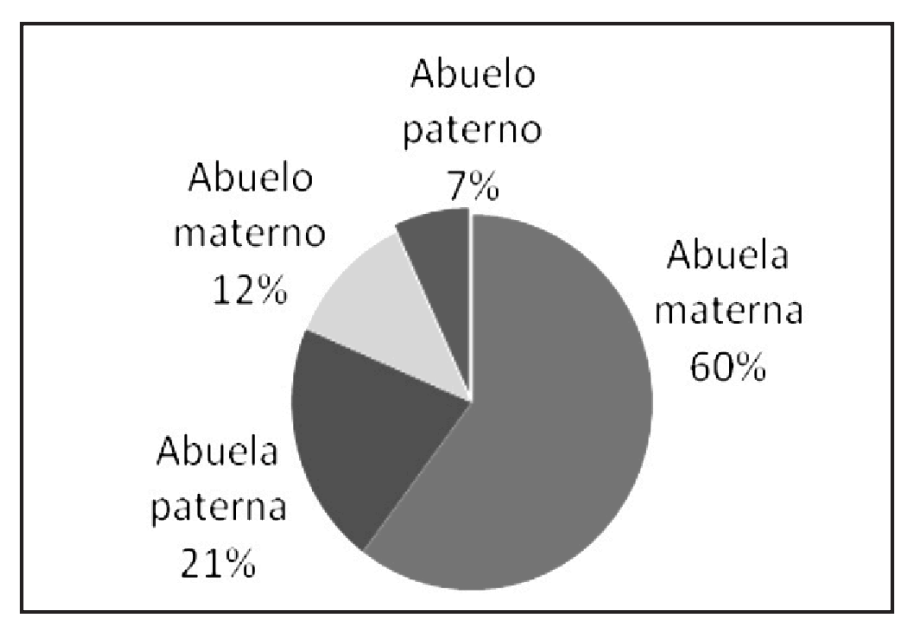

Gráfico 3. Porcentajes de abuelos favoritos según su familia de origen.

En diversos contextos la afinidad de los nietos con la abuela materna se inicia cuando estas ayudan a sus hijas adultas en la transición a la maternidad, y luego en el cuidado del pequeño. Los nietos tienden a estar más cercanos con los abuelos de la línea materna que con los abuelos de la línea paterna, quizás porque los primeros se involucran más que los segundos llegando a desempeñar un rol significativo en la vida de los nietos (Roberto y Stroes, 1992; Van Ranst, Verschueren y Marcoen, 1995; Smith, 1995; Emick y Hayslip, 1996; Triadó, Martínez y Villar, 2000; Block, 2000; Rico, Serra y Viguer, 2001).

\section{Ubicación geográfica.}

Kennedy (1992), señala que los nietos identifican como abuelos favoritos a aquellos que viven en la misma ciudad o a poca distancia, lo cual favorece una cercanía emocional, la sensación de un conocimiento mutuo y la influencia de los abuelos sobre los nietos.

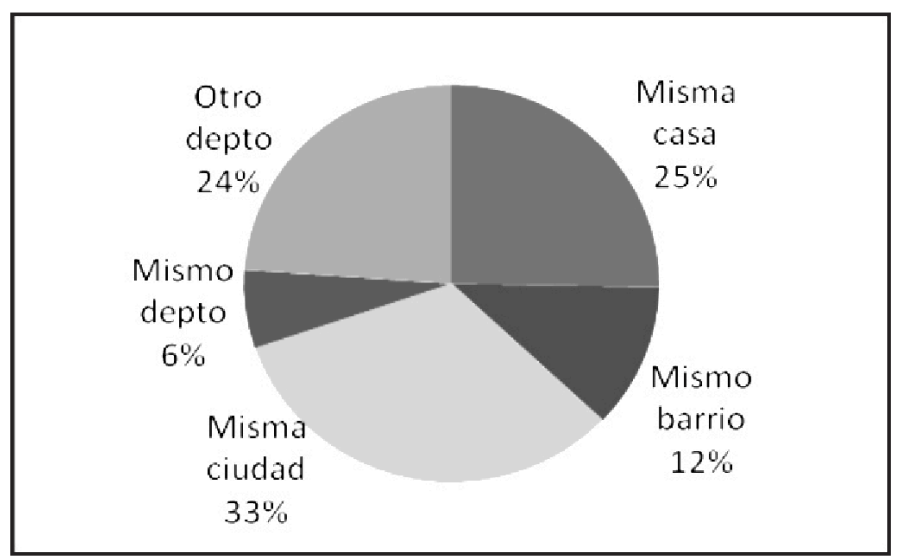

Gráfico 4. Porcentajes de la ubicación geográfica de los abuelos favoritos respecto a sus nietos.

Estos hallazgos de Kennedy se ratifican con los encontrados en este estudio, pues en Armenia en mayor medida los abuelos favoritos viven en la misma ciudad que sus nietos $32.7 \%$, en un $25.3 \%$ viven en la misma casa, en un $23.9 \%$ en otro departamento, en un $11.8 \%$ en el mismo barrio y el porcentaje más bajo $6.3 \%$ se da en los abuelos que viven en otros municipios, dentro del mismo departamento del Quindío.

\section{Frecuencia de contacto.}

Esta es otra característica, no menos importante y que ha sido estudiada en investigaciones anteriores. Es más frecuente en Armenia que el contacto de los nietos con sus abuelos favoritos sea de casi todos los días (47.4\%), seguido por los fines de semana (22.4\%), en menores proporciones se ven en vacaciones únicamente $(21 \%)$ y al menos una vez al mes (6.5\%), mientras el número de nietos que aseguran nunca ver a sus abuelos favoritos es mínimo (2.7\%).

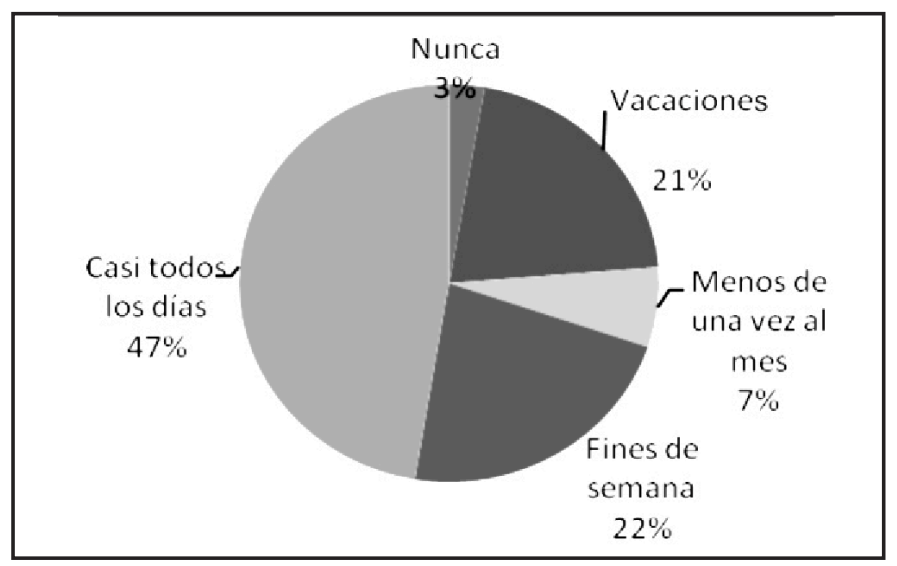

Gráfico 5. Porcentajes de frecuencia de contacto entre abuelos favoritos y nietos. 
La alta frecuencia de contacto, y por consecuencia el gran número de intercambios, potencia los efectos positivos en la calidad de las relaciones entre los abuelos y los nietos (Osuna, 2006). Ésta a su vez es determinada por la frecuencia de los encuentros de ambos, la relación cercana y frecuente entre abuelos y padres, las actividades de acompañamiento y la percepción de los abuelos como cuidadores y maestros (Kennedy, 1992).

\section{Ocupación del abuelo.}

La mayoría de los abuelos favoritos en Armenia están jubilados o sin trabajo remunerado (72.8\%), mientras un porcentaje mucho más bajo aún trabaja (27.2\%). Estos resultados coinciden con los hallados por Rico, Serra y Viguer (2001) quienes también encontraron que es más frecuente que los niños elijan como abuelos favoritos a aquellos que no trabajan o que son amas de casa, en el caso de las abuelas.

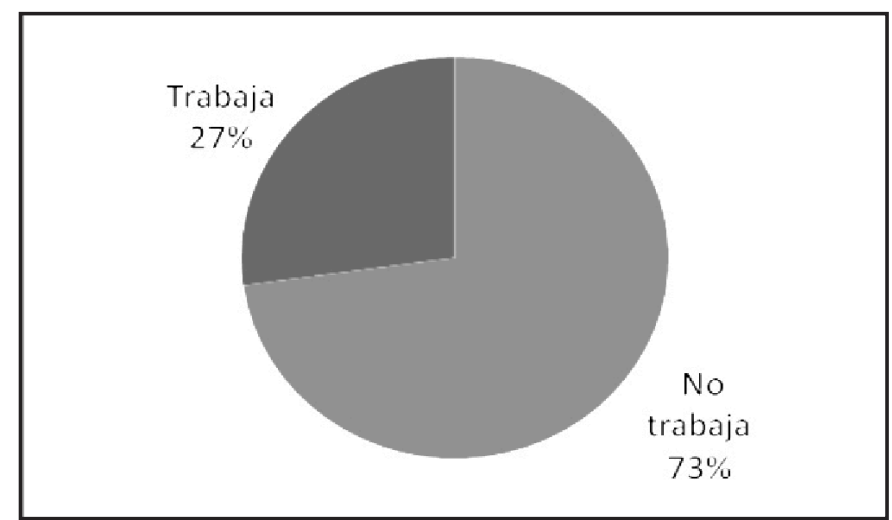

Gráfico 6. Porcentajes de ocupación de los abuelos favoritos.

Cabe anotar que es significativo el porcentaje de abuelos quindianos que aún trabajan. Hecho este que puede estar relacionado con los bajos niveles de desarrollo socioeconómico y la legislación en materia de seguridad social con que cuenta el país, pues un gran número de adultos mayores deben seguir laborando para su sustento y el de sus familias.

Estos hallazgos adquieren mayor importancia al considerar que cuando abuela y abuelo no trabajan, tienen más tiempo para dedicar al cuidado de sus nietos y posibilidades de una frecuencia de contacto mucho mayor con los niños, lo cual puede entablar una buena relación con ellos e influir de manera positiva en su proceso de socialización (Rico, Serra y Viguer, 2001).

El ser abuelo es un suceso normativo, que según Neugarten B,L. y Neugarten, D.A. (1987), ocurre en un momento determinado de la vida, el cual viene dado por factores exógenos caracterizando así el rol de abuelo 
más jóvenes. Es probable que las diferencias en la relación debidas al orden de nacimiento del nieto estén relacionadas con la edad del abuelo pues al nacimiento del primer nieto el abuelo es más joven (Kennedy 1992). Esta singularidad puede explicarse a través de los hallazgos de Bengtson (1985), Aldous (1995), Triadó, Martínez y Villar (2000), y Rico, Serra y Viguer (2001), quienes concluyeron que a medida que avanza la edad de los abuelos, disminuye su implicación con los nietos, se limita el contacto, se dejan de ejercer ciertos roles, actuando menos como guías familiares y más como conciliadores.

Tabla 4. de contingencia orden de nacimiento - edad abuelo favorito

En cuanto a la estructura familiar, se observa en la tabla que para todos los rangos de edad de los abuelos favoritos la estructura familiar que prevalece es la biparental. No obstante, los nietos que viven con otra persona, que normalmente es un abuelo, tienden a elegir como favoritos en mayor proporción a los abuelos menores de 60 años, mientras que los niños que pertenecen a una estructura biparental y monoparental tienen como favoritos a los abuelos entre 60 y 70 años.

\begin{tabular}{|l|c|c|c|}
\hline \multirow{2}{*}{$\begin{array}{c}\text { Características } \\
\text { del nieto }\end{array}$} & \multicolumn{3}{|c|}{$\mathrm{X}^{2}$} \\
\cline { 2 - 4 } & Valor & $\mathrm{Gl}$ & Sig. \\
\hline Edad del nieto & .919 & 2 & .632 \\
\hline Género del nieto & .100 & 1 & .752 \\
\hline Orden de nacimientos & 1.718 & 2 & .424 \\
\hline Número de hermanos & 1.043 & 2 & .593 \\
\hline Estructura familiar & 3.361 & 2 & .186 \\
\hline Ocupación de la madre & .235 & 1 & .628 \\
\hline
\end{tabular}

Tabla 5. de contingencia estructura familiar - edad abuelo favorito 

madres trabajadoras en el cuidado de los nietos; datos que no se observan en la población quindiana.

Distancia geográfica. Se encuentran diferencias significativas en la distancia geográfica que existe entre la diada en función del orden de nacimiento, el número de hermanos, la estructura familiar del nieto y la ocupación de la madre (tabla10 ). La distancia geográfica está estrechamente relacionada con la frecuencia de contacto pues, cuanto menos disten unos de otros, la frecuencia de contacto es mayor y, por ende la cercanía emocional. Se suman a esta dinámica factores como el número de nietos, si estos viven sólo con la madre y si esta última trabaja o no (Smith,1995; Kennedy, 1992).
Tabla 10. Relación de la distancia geográfica en función de las características del nieto

\begin{tabular}{|c|c|c|c|c|}
\hline \multirow{2}{*}{$\begin{array}{c}\text { Características } \\
\text { del nieto }\end{array}$} & \multicolumn{3}{|c|}{$\mathrm{X}^{2}$} & $\begin{array}{c}\text { U de Mann } \\
\text { Whitney }\end{array}$ \\
\cline { 2 - 5 } & Valor & gl & Sig. & Sig. \\
\hline Edad del nieto & 5.025 & 8 & .755 & \\
\hline $\begin{array}{c}\text { Género del nieto } \\
\text { Erden de } \\
\text { nacimiento }\end{array}$ & 2.185 & 4 & .702 & \\
\hline $\begin{array}{c}\text { Número de } \\
\text { hermanos }\end{array}$ & 21.648 & 8 & $.006^{* *}$ & $.026^{*}$ \\
\hline Estructura familiar & 89.882 & 8 & $.000^{* *}$ & $.000^{* *}$ \\
\hline Ocupación madre & 11.932 & 4 &. $\mathbf{0 1 8 *}$ & \\
\hline
\end{tabular}

Tabla 11. Contingencia orden de nacimiento - distancia geográfica

\begin{tabular}{|c|c|c|c|c|c|c|}
\hline \multirow[b]{2}{*}{ Orden nacimiento } & \multicolumn{6}{|c|}{ Distancia geográfica } \\
\hline & $\begin{array}{l}\text { En la misma } \\
\text { casa }\end{array}$ & $\begin{array}{l}\text { En el mismo } \\
\text { barrio }\end{array}$ & $\begin{array}{l}\text { En la misma } \\
\text { ciudad }\end{array}$ & $\begin{array}{c}\text { En el mismo } \\
\text { departamento }\end{array}$ & $\begin{array}{c}\text { En otro } \\
\text { departamento }\end{array}$ & Total \% \\
\hline Primer hijo & 33.9 & 9.7 & 25.6 & 47.4 & 22.9 & 100 \\
\hline Segundo hijo & 21.4 & 16.2 & 37.7 & 26.3 & 23.3 & 100 \\
\hline Tercer o más orden & 16.7 & 9.3 & 40.7 & 26.3 & 26.7 & 100 \\
\hline Total \% & 100 & 100 & 100 & 100 & 100 & \\
\hline
\end{tabular}

La tabla de contingencia indica la tendencia de los nietos de segundo y tercer o más orden de nacimiento a elegir como favoritos a los abuelos que viven en la misma ciudad, mientras que los nietos de primer orden eligen principalmente a los abuelos que viven en la misma casa.

el rol de abuelidad a edades más tempranas (Szinovacz, 1998), y paralelamente ha ido aumentando el número de mujeres madres solteras o inmersas en el mundo laboral que demandan el apoyo de sus padres en el cuidado de los hijos (Ruiz, 2008).

Los nietos que son hijos únicos o primogénitos están más cercanos a sus abuelos que los de otros órdenes de nacimiento (Kennedy, 1992). El hecho que los primogénitos elijan mayoritariamente a abuelos que viven en su misma casa puede atribuirse a que estos últimos participan en el cuidado de los nietos o por el contrario, es el abuelo quien requiere del cuidado de su familia. Pero la segunda opción es menos probable, dado que cada vez se ejerce

La tendencia de los preadolescentes según su número de hermanos, tiene una estrecha similitud con los datos anteriores; como se puede observar en la tabla de contingencia. Es más probable que los nietos que son hijos únicos tengan como favoritos a los abuelos que viven en la misma casa, mientras que los nietos que tienen uno, dos o más hermanos, tienden a elegir como favoritos a los abuelos que viven en la misma ciudad.

Tabla 12. Contingencia número de hermanos - distancia geográfica

\begin{tabular}{|c|c|c|c|c|c|c|}
\hline \multirow[b]{2}{*}{ Número de hermanos } & \multicolumn{6}{|c|}{ Distancia geográfica } \\
\hline & $\begin{array}{l}\text { En la misma } \\
\text { casa }\end{array}$ & $\begin{array}{l}\text { En el mismo } \\
\text { barrio }\end{array}$ & $\begin{array}{l}\text { En la misma } \\
\text { ciudad }\end{array}$ & $\begin{array}{c}\text { En el mismo } \\
\text { departamento }\end{array}$ & $\begin{array}{c}\text { En otro } \\
\text { departamento }\end{array}$ & Total \% \\
\hline Hijo único & 45.8 & 5.1 & 18.6 & 11.9 & 18.6 & 100 \\
\hline Un hermano & 23.1 & 13.0 & 37.5 & 28.9 & 24.0 & 100 \\
\hline Dos o más hermanos & 22.6 & 12.2 & 56.8 & 52.6 & 56.4 & 100 \\
\hline Total \% & 100 & 100 & 100 & 100 & 100 & \\
\hline
\end{tabular}


Respecto a la estructura familiar, es más probable que los nietos que viven con un abuelo o abuela, este sea el favorito; tal tendencia se mantiene cuando el nieto pertenece a una estructura monoparental. Pero cuando el nieto vive con ambos padres, tiende a elegir en mayor proporción a los abuelos que viven en la misma ciudad. Se confirma así el papel que juega el abuelo en familias monoparentales, donde según Rico, Serra y Viguer (2001), Emick y Hayslip (1996), Bengtson (1985) y Wilson (1986), bajo estas circunstancias aumenta la implicación directa de los abuelos, en especial los de la línea materna, participando de manera activa en la crianza y en diversas formas de apoyo hacia el nieto y el grupo familiar (Ruíz, 2008; Pinazo, 2003).

Tabla 13. Contingencia estructura familiar - distancia geográfica

\begin{tabular}{|c|c|c|c|c|c|c|}
\hline \multirow[b]{2}{*}{ Estructura familiar } & \multicolumn{6}{|c|}{ Distancia geográfica } \\
\hline & $\begin{array}{c}\text { En la misma } \\
\text { casa }\end{array}$ & $\begin{array}{l}\text { En el mismo } \\
\text { barrio }\end{array}$ & $\begin{array}{l}\text { En la misma } \\
\text { ciudad }\end{array}$ & $\begin{array}{c}\text { En el mismo } \\
\text { departamento }\end{array}$ & $\begin{array}{c}\text { En otro } \\
\text { departamento }\end{array}$ & Total \% \\
\hline Biparental & $\begin{array}{ll}32.0 & 13.9 \\
\end{array}$ & $\begin{array}{ll} & 15.3 \\
75.7 & \\
\end{array}$ & $\begin{array}{|ll|} & 36.7 \\
66.5 & \\
\end{array}$ & $\begin{array}{|ll|}73.7 & 8.1 \\
\end{array}$ & 26.0 & 100 \\
\hline Monoparental & 34.8 & 24.3 & 29.8 & 26.3 & 33.1 & 100 \\
\hline Con otra persona & 72.7 & 0.0 & 15.9 & 0.0 & 11.4 & 100 \\
\hline Total \% & 100 & 100 & 100 & 100 & 100 & \\
\hline
\end{tabular}

Si bien es mayor el porcentaje de madres que no trabajan, cuando estas lo hacen los nietos eligen con mayor frecuencia (31.5\%) a los abuelos que viven en la misma casa, por ser quienes se encuentran geográficamente más cerca, es más frecuente el contacto y por tanto la cercanía emocional, al tiempo que los abuelos contribuyen al cuidado mientras la madre cumple su jornada laboral. Incluso puede constituir una forma mutua de prestación de servicios en esta relación bidireccional, pues mientras los abuelos (principalmente la abuela, por ser quien más contribuye a la educación) aportan compañía, colaboran con las tareas del hogar y se ocupan de los niños, reciben a cambio los beneficios de estar en el grupo familiar a edades avanzadas (Pebley y Rudkin, 1999).

Tabla 14. Contingencia situación laboral de la madre - distancia geográfica

\begin{tabular}{|c|c|c|c|c|c|c|}
\hline \multirow{2}{*}{$\begin{array}{l}\text { Situación laboral } \\
\text { de la madre }\end{array}$} & \multicolumn{6}{|c|}{ Distancia geográfica } \\
\hline & $\begin{array}{c}\text { En la misma } \\
\text { casa }\end{array}$ & $\begin{array}{c}\text { En el mismo } \\
\text { barrio }\end{array}$ & $\begin{array}{c}\text { En la misma } \\
\text { ciudad }\end{array}$ & $\begin{array}{c}\text { En el mismo } \\
\text { departamento }\end{array}$ & $\begin{array}{c}\text { En otro } \\
\text { departamento }\end{array}$ & Total \% \\
\hline No trabaja & 19.8 & 11.6 & $\begin{array}{|ll|}67.9 & 36.1 \\
\end{array}$ & $62.2 \quad 6.3$ & 26.2 & 100 \\
\hline Trabaja & 31.5 & 13.0 & 32.1 & 37.8 & 31.7 & 100 \\
\hline Total \% & 100 & 100 & 100 & 100 & 100 & \\
\hline
\end{tabular}

En contraste, es más usual que cuando la madre no trabaja, los nietos tengan como favoritos a los abuelos que viven en la misma ciudad (36.1\%), quizás porque la madre puede ocuparse de los niños y no necesita de una intervención directa de los abuelos en la crianza y menos aún que vivan en la misma casa; pero no se puede obviar la cercanía geográfica que indiscutiblemente favorece la relación diádica en aquellos casos donde esta es positiva. De igual manera, una lectura vertical de la tabla 14 , permite observar que cuando las madres trabajan se dan mayores porcentajes de elección de abuelos cercanos y menores porcentajes de abuelos distantes. 

Tabla 18. Contingencia estructura familiar - frecuencia de contacto

\begin{tabular}{|c|c|c|c|c|c|c|}
\hline \multirow{2}{*}{ Estructura familiar } & \multicolumn{7}{|c|}{ Distancia geográfica } \\
\cline { 2 - 7 } & Nunca & $\begin{array}{c}\text { En vacaciones } \\
\text { únicamente }\end{array}$ & $\begin{array}{c}\text { Al menos una } \\
\text { vez al mes }\end{array}$ & $\begin{array}{c}\text { Fines de } \\
\text { semana }\end{array}$ & $\begin{array}{c}\text { Casi todos } \\
\text { los días }\end{array}$ & Total \% \\
\hline Biparental & 2.3 & 21.8 & 6.6 & 28.1 & $\mathbf{4 1 . 3}$ & 100 \\
\hline Monoparental & 3.0 & 22.7 & 6.9 & 16.3 & $\mathbf{5 1 . 2}$ & 100 \\
\hline Con otra persona & 4.5 & 6.8 & 4.5 & 4.5 & $\mathbf{7 9 . 5}$ & 100 \\
\hline
\end{tabular}

Esta misma tendencia es señalada por otros autores como Aldous (1995), Rubio y Garrido (1995), Block (2000) y Triadó y Villar (2000), para quienes además de que abuelos y nietos se puedan ver casi todos los días, desempeña un importante papel el hecho que disten poco uno del otro, que los abuelos pertenezcan a la línea materna y el nieto a una estructura monoparental, conllevando estos diversos aspectos a una relación más afín, de constante apoyo y de influencia del abuelo en el desarrollo social, (López y Cantero, 1999; Pineda y Ruiz, 1996), afectivo (López y Cantero, 1999), cognitivo y moral del nieto (Kohlberg, 1992).

\section{CONCLUSIONES}

La relación abuelo-nieto, articulada entre otras por la transmisión intergeneracional, está mediada por características específicas de abuelos y nietos, así como por el sistema familiar y social. Dado el vínculo bidireccional, con efectos tanto positivos como negativos, los abuelos se involucran cada vez más en el desarrollo y cuidado de sus nietos, adaptando las funciones de su rol a las necesidades, al momento evolutivo de ambos y a expectativas familiares, teniendo que cumplir la norma de no interferencia.

Las modificaciones acaecidas en el entorno donde se desarrolla la relación alteran la concepción de los roles de abuelo y nieto, obligando a su redefinición, a un nuevo ajuste al entorno cambiante reforzando las características especiales de afectuosidad, complicidad y satisfacción, surgidas en la interacción.

Al concluir el presente trabajo se constata que, para comprender con detenimiento la relación abuelos-nietos, se hace preciso estudiar la vida cotidiana de las personas. Volviendo la mirada hacia las interacciones intergeneracionales, podemos dar cuenta de las características de la figura del abuelo favorito, propias de su cultura y su momento histórico.
El género es una variable que establece diferencias en el desempeño del rol de abuelo y en su percepción. Confrecuencia, son las abuelas quienes están más próximas a sus nietos, comparten más actividades, intimidad y afecto, mientras que los abuelos son más distantes emocionalmente. A las abuelas se les atribuye roles de afectividad y cuidado, esto se refleja en la elección de los nietos, pues más del $80 \%$ de los preadolescentes encuestados señalan a las abuelas como favoritas.

Los nietos sienten mayor afinidad por los abuelos de la línea materna, quienes juegan un papel significativo en sus vidas, el contacto es más frecuente y la distancia geográfica más corta. La díada comparte actividades cotidianas y de esparcimiento que afianzan la relación, a la vez que los nietos adjudican a sus abuelos imágenes principalmente positivas.

Por otro lado, en lo que respecta a la investigación empíricoanalítica, se reconoce su utilidad como una metodología que permite, a partir de la percepción de los preadolescentes, abordar la relación abuelos-nietos, con el fin de construir conocimiento sistemático aplicable tanto dentro del área de la Psicología Evolutiva como de la Gerontología, y otras ciencias sociales y humanas.

Al emplear muestras compuestas por un número similar de hombres y mujeres, se evita caer en el error de generalizar a la población femenina resultados hallados a partir de muestras conformadas únicamente por hombres, a la vez que permite evidenciar tanto similitudes como diferencias entre géneros.

Por último, cabe señalar que los resultados de la investigación generan información que pueden aprovechar tanto los programas de proyección social de la Universidad del Quindío, como las entidades del Estado, las organizaciones privadas, y no gubernamentales, para la aplicación de proyectos con familias y comunidades. 


\section{BIBLIOGRAFÍA}

Aldous, J. (1995). "New Views of grandparents in intergenerational context". En Journal of family issues, 16(1), 104-122. Asociación de Gerontólogos del Quindío, AGQ - Secretaría del Interior y Desarrollo Social del Departamento. Hacia una política de vejez y envejecimiento: Quindío un departamento para todos las edades. Armenia, 2009.

Baumrind, D. (1971). "Effective parenting during the early adolescent transition”. En P.A. Cowan \& E.M. Hetherington (Eds.), Advances in family research, (2). NJ: Erlbaum.

Belsky, J. (1996). Psicología del Envejecimiento. Teoría, investigaciones e intervenciones. Barcelona: Masson.

Bengtson, V.L. (1985). "Diversity and symbolism in grandparental roles". En V.L. Bengtson y J.F. Robertson (Eds.), Grandparenthood. Beverly Hills: Sage publications.

Block, C. (2000). "Dyadic and gender differences in perceptions of the grandparent-grandchildren relationship". En International journal aging and human development, 51 (2), 85-104.

Castañeda, J. y cols (2004). "Cómo perciben los nietos adultos las relaciones con sus abuelos". En Anuario de Psicología, 35(1),107-123.

Cedrán, M. (2004). "Relaciones intergeneracionales no normativas: la relación de los nietos con sus abuelos afectados por una enfermedad neurodegenerativa". En Revista Multidisciplinar de Gerontología, 14(5), 262-268.

Cherlin, A. y Furstenberg, F. (1985). "Styles and strategies of grandparenting". En Bengtson y Robertson (Eds.), Grandparenthood. Beverly Hills: Sage publications.

Clark-Stewart, K.A. (1978): "And daddy makes three: the father's impact on mother and young child". En Child development, (49), 466-478.

Creasey, G. y Kaliher, G. (1994). "Age differences in grandchildren's perceptions of relations with grandparents". En Journal of Adolescence, (17), 411-426.

Dunifon, R. y Kowalesky-Jones, L. (2007). “The influence of grandparents in single-mother families”. En Journal of Marriage and Family, 69(2), 465-481.

Emick, M. y Hayslip, B. (1996). “Custodial grandparenting: new roles for middle-aged and older adults”. En International journal aging and human development, 43(2), 135-154.

Fernández-Ballesteros, R. (2001). "Environment conditions, health and satisfaction among the elderly: some empirical results". En Psicothema, (13), 40-49.

Hoff, A. (2007). "Patterns of intergenerational supportin grandparent-grandchild and parent-child relationships in Germany". En Ageing y Society, (27), 643-665.

Hurme, H. (1997). "Cross-cultural differences in adolescents' perceptions of their grandparents". En International journal aging and human development, 44(2), 221-253.

Jendrek, M.P. (1994). “Grandparents who parent their grandchildren: circumstaces and decisions”. En Gerontologist, (34), 206216.

Kahana, B. y Kahana, E. (1970). "Grandparenthood from the perspective of the developing grandchildren". En Developmental psicology, (3), 98-105.

Kennedy, G. (1992). "Quality in Grandparent/Grandchildren relationship". En International journal aging and human development, 35(2), 83-98.

Kennedy, G. (1992). "Shared activities of grandparents and grandchildren". En Psychological reports, (70), 211-227.

Kohlberg, L. (1992). Psicología del desarrollo moral. Barcelona: Descleé de Brouwer.

López, F. y Cantero, M.J. (1999). "La intervención en la familia”. En F. López; I. Etxebarria; M.J. Fuentes y M.J. Ortiz (Eds.), Desarrollo Afectivo y Social. Madrid: Pirámide.

Mckay, V.C. y Caverly, R.S. (1995). "Relationships in later life: the nature of inter-and intragenerational ties among grandparents, grandchildren, and adult siblings". En Nussbaum y Coupland (Eds.), Handbook of comunication and aging research. New Yersey: Lawrence Erlbaum associates.

Monserud, M. (2008). "Intergenerational relationships and affectual solidarity between grandparents and young adults". En Journal of Marriage and Family, 7(1), 182-195.

Musitu, G. y Cava, M.J. (2001). La familia y la educación. Barcelona: Octaedro.

Neugarten, B.L. (1964). Personality in middle and late life. New York: Atherton.

Neugarten, B. y Weistein, K. (1964). "The changing American grandparent". En Journal of marriage and the family, (26), 199204.

Neugarten, B,L. y Neugarten, D.A. (1987). “The changing meanings of age”. Psiychology Today, 21, 29-33. 
Ochaita, E. y Espinosa, M.A. (1995). "Nuevas relaciones en el seno de las familias de finales del siglo XX: las relaciones entre miembros de familias alternas". En Infancia y sociedad, (29), 27-46

Ong, D. y Quah, S. (2007). “Grandparenting in divorced families”. En Singapore Journal of Legal Studies, 25-50.

Osuna, M. J. (2006). "Relaciones familiares en la vejez: vínculos de los abuelos y de las abuelas con sus nietas en la infancia”. En Revista Multidisciplinar de Gerontología, 16(1), 16-25.

Parke, R.D. y Tinsley, B.J. (1981): "The father's role in infancy: determinants of involvement in caregiving and play". En M.E. Lamb (Ed.), The role of the father in child development. New York: Wiley.

Pebley, A. y Rudkin, L.(1999). “Grandparents caring for grandchildren”. En Journal of family issues, 20(2), 218-242.

Pinazo, S. (1999). "Influencia de los abuelos en la socialización familiar de los nietos. Revisión de la literatura científica”. En Revista española de Geriatría y Gerontología, 34(4), 231-236.

Pinazo, S. (1999). "Significado social del rol de abuelo". En Revista Multidisciplinar de Gerontología, (9), 169-176.

Pinazo, S. (2003). "Impacto psicosocial del acogimiento familiar en familia extensa: el caso de las abuelas y los abuelos acogedores". En Revista Multidisciplinar de Gerontología, 13(2), 89-101.

Pineda, O.M. y Ruiz, M.C. (1996). Estilos de Socialización de los Abuelos. Armenia: Asculquin.

Power, T.G. y Parke, R.D. (1982). "Play as a context for early learning: lab and home analysis". En Siegel, I.E. y Laosa, L.M. (Eds.), The family as a learning environment. New York: Plenum.

Procuraduría General de la Nación. Huérfanos de padres vivos. Armenia, 2009.

Rico, C; Serra, E. y Viguer, P. (2001). Abuelos y Nietos. Abuelo favorito-abuelo útil. Madrid: Pirámide.

Roberto, K. y Stroes, J. (1992). "Grandchildren and granparents: roles, influences, and relationships". En International journa I aging and human development, 34(3), 227-239.Rubio, R.

Rodrigo, M. y Palacios, J. (1998). Familia y Desarrollo Humano. Madrid: Alianza.

y Garrido, J.A. (1995). "Actividad social y redes sociales en relaciones intergeneracionales abuelos/as-nietos/as". En Infancia y sociedad, (29), 164-181.

Ruiz, D. (2008). "The changing roles of African American grandmothers raising grandchildren: An exploraty study in the Piedemont Region of North Carolina". En Western Journal of Black Studies, 32(1), 62-71.

Sáez, N.; Rubio, R. y Dosil, A. (1996). Tratado de Psicogerontología. Valencia: Promolibro.

Secretaría de Salud Municipal. "Armenia saludable, un compromiso de todos". En Plan de Salud Territorial. Armenia, 20082011.

Smith, P.K. (1995). "Grandparenthood". En Bornstein, M. (Ed.), Handbook of parenting. Status and social conditions of parenting. New Jersey: Lawrence Erlbaum Associates.

Smith, P.K. (1991). The psychology of grandparenthood. An international perspective. London: Routledge.

Spera, C. y Matto, H. (2007). "A Contextual-Congruence Model of Socialization". En Families and Society, 38(4), 551-560.

Strom, R. y Strom, S. (1997). "Building a theory of grandparent development". En International journal aging and human development, 45(4), 255-286.

Szinovacz, M. (1998). "Grandparents today's: a demographic profile". En The gerontologist, 38(1), 37-52.

Triadó, C. y Villar, F. (2000). "El rol de abuelo: cómo perciben los abuelos las relaciones con sus nietos". En Revista Española de Geriatría y Gerontología, 35(S2), 30-36.

Triadó, C.; Martinez, G. y Villar, F. (2000). "El rol y la importancia de los abuelos para sus nietos adolescentes". En Anuario de psicología, 31(2), 107-118.

Van Ranst, N.; Verschueren, K. y Marcoen, A. (1995). "The meaning of grandparents as viewed by adolescent grandchildren: an empirical study in Belgium". En International journal aging and human development, 41(4), 311-324.

Viguer, P. (1996). La infancia urbana de los 90: cambios en el estilo de vida debidos a la incorporación progresiva de la mujer al mundo laboral. Universidad de Valencia: Tesis Doctoral.

Wachs, T.D. y Gruen, G.E. (1982). Early experience and human development. New York: Plenum

Wilson, M.N. (1986). "The black extended family: an analytical consideration". En Developmental Psychology, (22), 246-256. 


\title{
Diseño de software educativo basado en competencias
}

\section{Design of educational software based on skill development}

\author{
Manuel Fernando Caro*, Raúl Emiro Toscano*, \\ Filadelfia María Hernandez**, María Elena David**
}

Recibido: Abril 8de 2009

Aceptado: Junio 1 de 2009

Correspondencia:Departamento de Informática, Universidad de Córdoba, Montería Córdoba. Email: loshigos@yahoo.es

\section{RESUMEN}

El presente artículo describe un modelo de diseño de software educativo basado en competencias, el cual presenta una visión integral del desarrollo de estas aplicaciones mediante la combinación de componentes pedagógicos, didácticos, multimediales y de ingeniería de software.

El modelo sugerido se compone de cinco fases que detallan paso a paso los aspectos a tener en cuenta para la creación de software educativo. La fase inicial constituye la descripción del diseño educativo, en la cual se analiza la necesidad educativa, se plantean los objetivos de aprendizaje y se describen las competencias que se pretenden desarrollar con la aplicación; del diseño de éstas resultan las siguientes subfases: diseño de contenidos, diseño pedagógico y diseño de aprendizaje. Las competencias son el aspecto fundamental que abarca el desarrollo de este modelo, las cuales son primordiales para la realización de las fases a seguir conformadas por el diseño computacional y el diseño multimedial, estas se encargan del análisis y modelado del software, y del sistema de comunicación hombre-máquina. En la fase de producción se ensamblan los componentes elaborados o recolectados, según el caso. La última fase es la de aplicación, donde se hacen las pruebas de rigor para evaluar el desempeño del software en los contextos para los que fue desarrollado.

Palabras Claves: Software educativo, ingeniería de software, educación, informática, competencias.

\begin{abstract}
This article describes an educational software design model based on skills development, which presents a vision of developing these applications by combining components of teaching, learning, multimedia and software engineering.

The suggested model consists of five stages that describe the steps to be taken into account for the creation of educational software. The initial phase is the description of education design, which analyzes the educational need, outlines the learning objectives, and summarizes the skills that the application look forward to develop. From the design of these aspects, the following sub-phases are developed: content design, pedagogical design and learning design. Skills are the key point that involves the development of this model, which are essential for carrying out the steps to follow the computational design and multimedia design; these are responsible for the analysis and software modeling, and for the man-machine communication system. In the production phase are assembled the components produced or collected, as appropriate. The last phase is implementation, where tests are applied to evaluate the rigor of the software in the contexts for which it was developed.
\end{abstract}

Keywords: Educational software, software engineering, education, computing, skills.

\section{INTRODUCCIÓN}

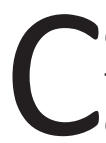
omo concepto de trabajo, desde la década del 90, el tema de competencias se ha convertido en una corriente de pensamiento dirigida al mejoramiento de la calidad de la educación, luego que el MEN (Ministerio de
Educación Nacional) con el sistema Nacional de evaluación de la calidad (SABER) lo estableciera con el decreto 1860 de 1994 y el decreto 230 de 2002, especialmente para el desarrollo de propuestas educativas y pedagógicas [1], muchas de las cuales tienen la tecnología como mediación.

\footnotetext{
*Grupo de Investigación Edupmedia. Departamento de Informática. Universidad de Córdoba. Colombia.

** Joven investigadora, grupo Edupmedia. Departamento de Informática. Universidad de Córdoba.
} 
Gracias a los adelantos que la modernidad y la innovación tecnológica han traído a todos los campos, los software educativos han tomado gran importancia en los procesos de enseñanza, siendo herramientas que aportan tanto al docente como al estudiante espacios dinámicos de aprendizaje; por lo cual, y haciendo eco a lo mencionado anteriormente, es necesario que el diseño de los mismos tengan como eje central de su diseño educativo el sistema de competencias a desarrollar, procurando hacer énfasis en capacitar a los alumnos para enfrentar los retos que se le puedan presentar en la sociedad actual, contribuyendo desde estas aplicaciones a la formación de personas con pensamiento crítico y analítico.

No obstante a lo anterior, en general los modelos de desarrollo de software educativos no están contextualizados a la realidad de la educación en Colombia, al no contener directrices claras acerca del manejo de competencias en su diseño, dándole más importancia a la ingeniería de software o al diseño multimedial. El modelo que EDUPMEDIA presenta, prioriza el desarrollo de competencias, sin dejar de lado la parte estructural.

El modelo de diseño de software educativo desarrollado por el grupo de Investigación EDUPMEDIA, surge de la sistematización de la experiencia que los docentes y jóvenes investigadores del grupo han tenido durante tres años en el desarrollo de software adaptado a la realidad educativa y basado en el desarrollo de competencias.

\section{ESTRUCTURA DEL MODELO}

\section{Competencias como eje central del modelo Edupmedia.}

A continuación se describen los aspectos que hacen especialmente particular este modelo que presenta el grupo de investigación EDUPMEDIA para el diseño de software educativo, cuyas características frente a los que en la actualidad se utilizan, brindan ventajas para su implementación en el contexto colombiano. Siendo las competencias el eje central del modelo, es necesario indicar las razones que lo justifican, con lo cual se brinda el sustento básico de su diferenciación frente a los modelos existentes así:

1) Los modelos utilizados en la actualidad no presentan una sección o apartado que brinde toda la información relacionada con las competencias requeridas según las necesidades educativas detectadas en la investigación previa, como sí lo contempla el modelo Edupmedia, pues en éste no sólo se enuncian las competencias, sino que también se establece un formato de presentación (por objetivos y normas) que agrupa todos los componentes de las misma, lo que permite al diseñador recoger los aspectos necesarios para realizar una documentación completa, lo cual guiará con mayor certeza el proceso de desarrollo.

2) El diseño del sistema de competencias actúa como eje integrador del proceso de diseño y producción del software educativo, pues cada una de sus secciones brinda información de entrada para otros procesos del desarrollo, convirtiéndose así en el centro de partida y principal fuente de información para las fases siguientes. Así pues, la sección de Recursos dentro de la Norma da nociones precisas acerca de las herramientas complementarias que requiere el estudiante para realizar las actividades propuestas, las cuales debe proveer el software educativo.

* La sección de los Elementos brinda las bases de las actividades que el usuario debe desarrollar con el software, por lo cual el diseñador debe tomar la decisión de hacer una ventana para cada elemento o agrupar varios elementos en una ventana; cabe destacar que a mayor cantidad de elementos por ventana, ésta se toma más compleja.

*El listado de conceptos que se obtienen de las competencias y que se consignan en la sección de los conceptos de origen al diseño de contenidos, en el cual se determina, el nivel de profundidad y el tipo de vocabulario que se utilizará en el software.

* La sección del concepto brinda al diseñador información que le permite determinar las características del ambiente que debe rodear las actividades de aprendizaje que se van a realizar con el software. Éstas pueden ser de tipo físico (laboratorios u otros lugares), o de tiempo (actualidad, siglo

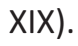

* La sección de los indicadores servirá para el diseño del sistema de evaluación del software educativo, pues en ellos se evidencian las habilidadesy conocimientos que debe demostrar una persona para la realización de una tarea (elemento) en particular dentro de la competencia. Con lo dicho anteriormente se evidencia que el eje central de todo el modelo son las competencias.

Por otra parte, desde lo pedagógico se puede aducir que el modelo EDUPMEDIA no se centra con ninguna teoría en particular, hecho que lo hace flexible y le permite amoldarse a las condiciones propias de la población objeto y a las características propias del entorno de la necesidad estudiada. También es factible que el modelo se adapte a las directrices pedagógicas propia de cada institución educativa, las cuales se encuentran consignadas en su Proyecto Educativo Institucional (PEI), así no se entraría a imponer un software que se trabaje en forma diferente a la realidad escolar. 


\section{Descripción general.}

El modelo de desarrollo de software educativo que se plantea en este escrito tiene como base fundamental el sistema de competencias, e integra cinco fases: Diseño educativo, diseño multimedial, diseño computacional, producción y aplicación. Este modelo es sencillo de aplicar y sus fases están bien documentadas, lo que lo hace idóneo para su utilización en procesos de desarrollo de software educativo.

Cada fase del modelo está bien delimitada y se encarga de un trabajo en particular así: en la Fase I se hace el estudio de los factores educativos que sustentarán el diseño del software en cuanto a la didáctica, la pedagogía y la ética. La Fase II, trata acerca de los aspectos estéticos y del sistema de comunicación, según la población, sistema de contenidos y estrategias planeadas el la fase anterior. En la Fase III se trabajan los fundamentos de ingeniería de software que requiere el desarrollo de una aplicación robusta y amigable. En la Fase IV se desarrollan y ensamblan los componentes del software, finalmente en la Fase $V$ se utiliza el software en el aula de clases, evaluando su pertinencia e impacto. A continuación se explican con más detalle cada fase.

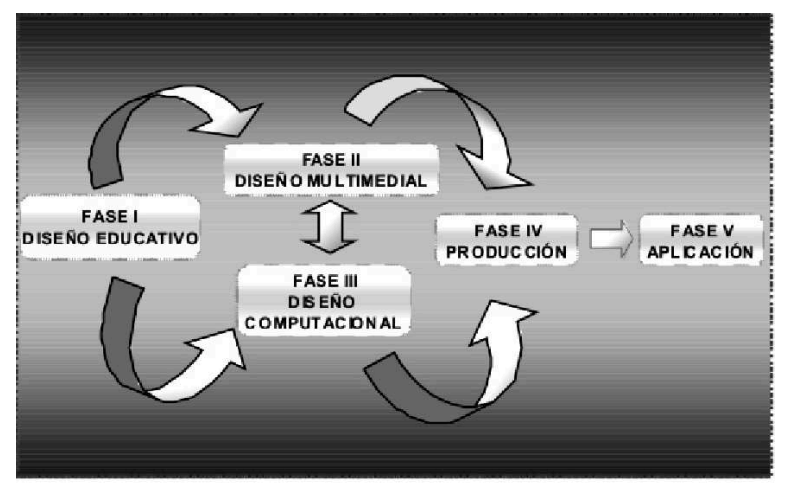

Figura 1. Modelo para el desarrollo de software educativo basado en competencias (Edupmedia-2008)

\section{Fase I: Diseño educativo.}

Comprendiendo las necesidades que se presentan en la educación con relación al proceso de enseñanza -aprendizaje, es necesario realizar un estudio exhaustivo sobre el aprendizaje educativo que conlleve al establecimiento de un plan de trabajo en donde se especifique los aspectos que comprendan las distintas etapas que hacen parte de la fase del diseño educativo. Estas etapas se definen a continuación:





Figura 2. Fase I. Diseño educativo. (Edupmedia-2008)

\section{Análisis de la Necesidad Educativa}

Al iniciar un proceso de diseño y desarrollo de software educativo es importante tener presente los problemas que se evidencian en una población determinada para saber exactamente el tipo de necesidad educativa en la que se va hacer énfasis, identificando las posibles causas que permitan plantear las diversas alternativas de solución a la problemática encontrada.

Entre las posibles fuentes de información podríamos contar los documentos de las instituciones educativas, folletos, boletines informativos, planes de estudio, estadísticas sobre pruebas de estado, libros de apuntes, entrevistas y cuestionarios.

\section{Diseño de Fines Educativo}

En el proceso de enseñanza y aprendizaje intervienen diversos factores que propenden la calidad educativa, dentro de estos factores sobresalen los fines educativos que integran los objetivos de aprendizaje, los valores y las dimensiones humanas, estos constituyen los propósitos fundamentales de la enseñanza que serán el punto de partida del desarrollo de las actividades escolares.

El diseño de fines educativos comprende el nivel cognitivo, el cual hace referencia al tipo de conocimiento que serán trabajados por los estudiantes y se redacta normalmente en forma de objetivos, y el nivel valorativo, el cual hace referencia a los valores, normas y conductas que serán privilegiadas [2].

\section{Diseño del Sistema de Competencias}

En el contexto educativo el término competencias se extiende al conjunto de actividades que se deben realizar para enfatizar el desarrollo de potencialidades del sujeto a partir de lo que aprende en la escuela [3]. Ahora bien, la educación en Colombia se rige por un sistema de competencias en donde se educa al estudiante para que éste sea activo en la sociedad, es decir que sea capaz 
de enfrentar las distintas situaciones que se le presenten de una forma crítica y responsable, permitiendo que se desenvuelva en un espacio social, orientado por un pensamiento de carácter sistémico y tecnológico. A partir de esto es necesario incluir en la creación de software educativo especificaciones claras acerca de cómo organizar las competencias que se pretenden desarrollar con el software. En este caso en la primera fase del modelo, se introduce una sección para gestionar las competencias que se han identificado, luego de realizar el análisis de la necesidad educativa. Estas se estructuran en un formato que comprende dos grandes ámbitos que son los objetivos y las normas, los cuales se describen a continuación:

\section{Objetivos}

Esta dimensión abarca el enunciado y los elementos de la competencia.

*Enunciado: Los objetivos son elementos fundamentales en todo proceso debido a que estos constituyen el derrotero a seguir y admite a su vez la evaluación del estudio abordado. En esta sección se establecerá los objetivos de las competencias para ampliar las nociones sobre la forma de definir un ambiente de aprendizaje. Aquí se indica las finalidades que se pretenden lograr o adquirir por parte de los sujetos involucrados en el proceso.

*Elementos: Para el tratamiento de las competencias se requieren una serie de actividades que dependen de cada objetivo, las cuales son enunciadas específicamente determinando lo que el estudiante debe ser capaz de realizar para cumplir el objetivo general.

\section{Norma}

Es la dimensión de la competencia que trata acerca del contexto, los recursos y las evidencias de conocimientos.

Contexto: Aquí se especifica el contexto donde se hace evidente la competencia, este puede ser un lugar físico o una situación determinada por un problema o reto que se debe solucionar.

Recursos: Son los recursos tangibles e intangibles que se requieren para realizar la competencia en el contexto determinado. Estos recursos se deben proveer en lo posible con el software. Así por ejemplo si se requiere un diccionario o una libreta de apuntes, el software debe proveer herramientas de diccionario y de libreta o agenda según el caso. Por tanto esta sección es el punto de partida para los elementos que debe llevar la barra de herramientas.
Indicadores: También llamados evidencias, son los conocimientos o actitudes que se deben ver o evaluar para saber si el estudiante o persona es competente. Por cada elemento de la competencia se pueden tener uno o más indicadores, por tanto esta sección será la base para la elaboración del sistema de evaluación del aprendizaje del software educativo.

\section{Conceptos}

Esta sección contiene una lista detallada de los conceptos que aparecen en los apartados anteriores del formato de competencias y que se relacionan directa o indirectamente con el ámbito de la temática del software educativo. Aquí no es necesario definir o explicar cada concepto, pues más adelante con esta lista se desarrolla el diseño de contenidos donde se tratan con mayor profundidad sus significados y relaciones.

\section{Habilidades Requeridas}

La realización de cualquier actividad implica ciertos conocimientos, destrezas que son necesarias para lograr su cumplimiento, esto conlleva a que los estudiantes deban poseer ciertas aptitudes que se requieren para desarrollar cada una de las competencias así:

Intelectuales: Las habilidades intelectuales son aquellas que se necesitan en la realización de actividades mentales. Los test de Coeficiente intelectual $(\mathrm{Cl})$, los test de admisión, los test de admisión para el postgrado en negocios, están diseñados para asegurarse de la habilidad intelectual de los individuos [4].

Las habilidades intelectuales como la destreza numérica, la comprensión verbal, la velocidad perceptual, el razonamiento inductivo, el razonamiento deductivo, la visualización espacial y la memoria, son las aptitudes que deben poseer los estudiantes como requisito para desarrollar las actividades propuestas en cada competencia.

Físicas: Las habilidades físicas son aquellas que se necesitan para realizar tareas que demandan vigor, destreza manual, fortaleza y características parecidas [4].

Las habilidades físicas comprenden las destrezas motoras que son fundamentales para el manejo de herramientas computacionales, que permiten a los estudiantes realizar con menor dificultad distintos tipos de actividades, en este caso los que intervienen en las competencias planteadas.

A Continuación se presenta un ejemplo del formato de cómo se diseña una competencia. 
Tabla 1. Ejemplo de Formato de competencias. (Software Lenguaje Maya - Edupmedia-2008)

\begin{tabular}{|c|c|}
\hline Competencia \#1 & Tipo: cognitiva \\
\hline Objetivos & Norma \\
\hline 1:Enunciado & 1: Contexto \\
\hline \multirow{3}{*}{$\begin{array}{l}\text { Reconocer palabras en lenguaje } \\
\text { maya de acuerdo a su simbología } \\
\text { basada en glifos. }\end{array}$} & $\begin{array}{l}\text { Cuando se le solicite escribir y conformar una } \\
\text { palabra en el lenguaje maya }\end{array}$ \\
\hline & 2: Recursos \\
\hline & $\begin{array}{l}\text { Libreta de apuntes cuadriculada, lápiz, } \\
\text { borrador, diccionario guía Maya }\end{array}$ \\
\hline 2: Elementos & 3:Evidencias \\
\hline \multirow[t]{3}{*}{$\begin{array}{l}\text { 1.Identificar los verbos y la } \\
\text { numeración del lenguaje maya }\end{array}$} & $\begin{array}{l}\text { 1.1 Reconoce la estructura de los verbos del } \\
\text { lenguaje maya (chum; ak'taj; tzak; chuk; jatz; } \\
\text { puluuy; uhti) }\end{array}$ \\
\hline & $\begin{array}{l}\text { 1.2 Reconoce el significado de los verbos del } \\
\text { lenguaje maya. (sentarse-chum; bailar-ak'taj; } \\
\text { conjurar-tzak; agarrar-chuk; golpear-jatz; } \\
\text { quemar-puluuy; suceder-uhti) }\end{array}$ \\
\hline & $\begin{array}{l}\text { 1.3 Identifica la numeración en maya del 1-10, } \\
\text { su escritura y pronunciación (JUN, KA, HUX, } \\
\text { KAN, JO, WAK, WUK, WAXAK, B'OLON, } \\
\text { LAJUN) }\end{array}$ \\
\hline \multirow[t]{3}{*}{$\begin{array}{l}\text { 2.Conocer los títulos y colores en } \\
\text { el lenguaje maya }\end{array}$} & $\begin{array}{l}\text { 2.1 Identifica los títulos de la lengua maya } \\
\text { (baahkab; k' inich; itz' aat;' a tz'ihb; 'a yuxul) }\end{array}$ \\
\hline & $\begin{array}{l}\text { 2.2 Identifica los colores del lenguaje } \\
\text { maya.(chak; ik; k' an; sak; yax) }\end{array}$ \\
\hline & $\begin{array}{l}\text { 2.3 Reconoce el significado de los títulos del } \\
\text { lenguaje maya. (pri mero del mundo (titulo de } \\
\text { militar)-baahkab; gran sol- k' inich; hombre } \\
\text { inteligente o artista-itz' aat; el de la escritura-'a } \\
\text { tz'ihb; el del tallado-'a yuxul) }\end{array}$ \\
\hline $\begin{array}{l}\text { 3. Identificar las diferentes } \\
\text { deidades de la lengua maya }\end{array}$ & $\begin{array}{l}\text { 3.1 Identifica las deidades del lenguaje maya } \\
\text { (Ah puch-Dios de la muerte violenta y } \\
\text { sacrificios humanos; Chac-Dios de la lluvia, y } \\
\text { fertilidad de la agricultura; Ek chuac-Dios de } \\
\text { la guerra; Hunab ku-El creador; Itzamna-Hijo } \\
\text { de Hunab Ku; Ixchel-protectora de las } \\
\text { parturientas y la inventora del Arte del Tejido; } \\
\text { Ix Tab-Diosa de los suicidas; Kukulcan-Dios } \\
\text { del viento; Xaman ek-Dios de la estrella polar; } \\
\text { Yun Kaax-Dios del maiz y la agricultura) }\end{array}$ \\
\hline
\end{tabular}




\section{Diseño de Contenidos}

Para la realización de un software educativo es necesario describir cada contenido que comprenda el problema estudiado, indicando la definición de cada categoría conceptual utilizada en dicho material educativo, haciendo énfasis en las características principales del conjunto de bases conceptuales.

El contenido se obtiene del listado de la sección de conceptos del formato de las competencias (ver tabla 1). Si existe más de una competencia, se hace un único listado con la sumatoria de todos los conceptos y se procede a elaborar sus definiciones según las características de la población y el nivel de profundidad deseado.

Tabla 2. Vista parcial de la matriz del diseño de contenidos. (Software Lenguaje Maya - Edupmedia-2008)

\begin{tabular}{|l|l|l|}
\hline Concepto & Características & Definición/Descripción \\
\hline Sentarse-chum & & Asumir el trono \\
& & \\
\hline Bailar-ak taj & & \\
& & \\
\hline
\end{tabular}

Diseño Pedagógico

La pedagogía se refiere al saber o discurso sobre la educación como proceso de socialización, de adaptación. En sentido estricto, por pedagogía entendemos el saber riguroso sobre la enseñanza, que se ha venido validando y sistematizando en el siglo XX como una disciplina científica en construcción, con su campo intelectual de objetos y metodologías de investigación propios, según cada paradigma pedagógico [5].

En esta sección se especifica en primera instancia el modelo pedagógico que se ha seleccionado, recordando que el modelo Edupmedia no trabaja con un modelo estándar, sino a partir de la elección hecha por los expertos teniendo en cuenta diversos factores como las competencias a desarrollar y las necesidades educativas específicas y el contexto donde se aplicará.

El modelo pedagógico seleccionado se tomará como base para el diseño de las actividades de aprendizaje que tendrá el software educativo, esto se hace en forma detallada para tener una visión amplia del modelo en cuestión. Una vez hecho eso, se procede a especificar las características o aspectos de dicho modelo que serán materializadas en el software, pues generalmente debido a la gran variedad de aspectos que abarca un modelo en particular, no todos son viables de aplicar a determinadas necesidades educativas.

\section{Diseño de Aprendizaje}

Una vez realizado el diseño pedagógico, se inicia el diseño de una estrategia de aprendizaje, cuyo propósito es otorgar a los estudiantes estrategias efectivas para el mejor desempeño en áreas y contenidos específicos. La utilización de distintas formas de aprendizaje dependerá de las actividades efectuadas y de las características cognitivas de los estudiantes.

Para realizar el diseño de aprendizaje se lleva a cabo el siguiente proceso:

Inicialmente se toman los elementos de la competencia seleccionados y sus indicadores (trabajado en la sección diseño de competencias), posteriormente se especifican las características del modelo pedagógico que se verán reflejadas en el software (trabajado en la sección de diseño pedagógico); con esos tres datos se diseña una secuencia de aprendizaje cuya finalidad es que los estudiantes realicen las actividades descritas en los elementos bajo las directrices especificadas en las características del modelo pedagógico. La secuencia debe evidenciar los aspectos relacionados en los indicadores, si dicha secuencia cumple con los aspectos mencionados, puede ser considerada como adecuada para continuar el proceso de diseño.

Tabla 3. Ejemplo resumido del diseño de la secuencia de aprendizaje basado en competencias. (Software Lenguaje Maya - Edupmedia-2008)

\begin{tabular}{|l|l|l|}
\hline \multicolumn{2}{|l|}{ COMPETENCIA \# 1} \\
\hline Elementos & Aplicación modelo pedagogía & Indicadores \\
\hline $\begin{array}{l}\text { 1.Identificar } \\
\text { los verbos y la } \\
\text { numeración del } \\
\text { lenguaje maya }\end{array}$ & $\begin{array}{l}\text { Conoce los verbos más significativos de } \\
\text { una nueva cultura. }\end{array}$ & $\begin{array}{l}\text { 1.1.Reconoce la } \\
\text { estructura de los } \\
\text { verbos del } \\
\text { lenguaje maya } \\
\text { (chum; ak taj; } \\
\text { tzak; chuk; jatz; } \\
\text { puluuy; wol; uhti; } \\
\text { pas; lok })\end{array}$ \\
\hline $\begin{array}{l}\text { Secuencia degidos son los principales aprendizaje } \\
\text { Se ilustran los verbos más importantes del lenguaje maya según la representación y } \\
\text { pronunciación en maya y la representación de estos en español. El usuario escogerá } \\
\text { libremente que verbo desea visualizar. Para determinar el indicador 1.1 se } \\
\text { mostrarán botones que representan los verbos, los cuales el usuario al darles clic, se } \\
\text { le mostrará la representación y pronunciación por medio de imágenes. }\end{array}$ \\
\hline
\end{tabular}

\section{Fase II: Diseño multimedial}

A partir de los resultados obtenidos de la fase del diseño educativo se establece la fase número II denominada diseño multimedial. Esta fase se encarga de la descripción de las etapas que permitirán conocer la planeación de actividades a desarrollar, utilizando representaciones gráficas y recursos multimedia que en conjunto mostrarán el diseño de cada interfaz y la estructura que se va a utilizar en el software. 


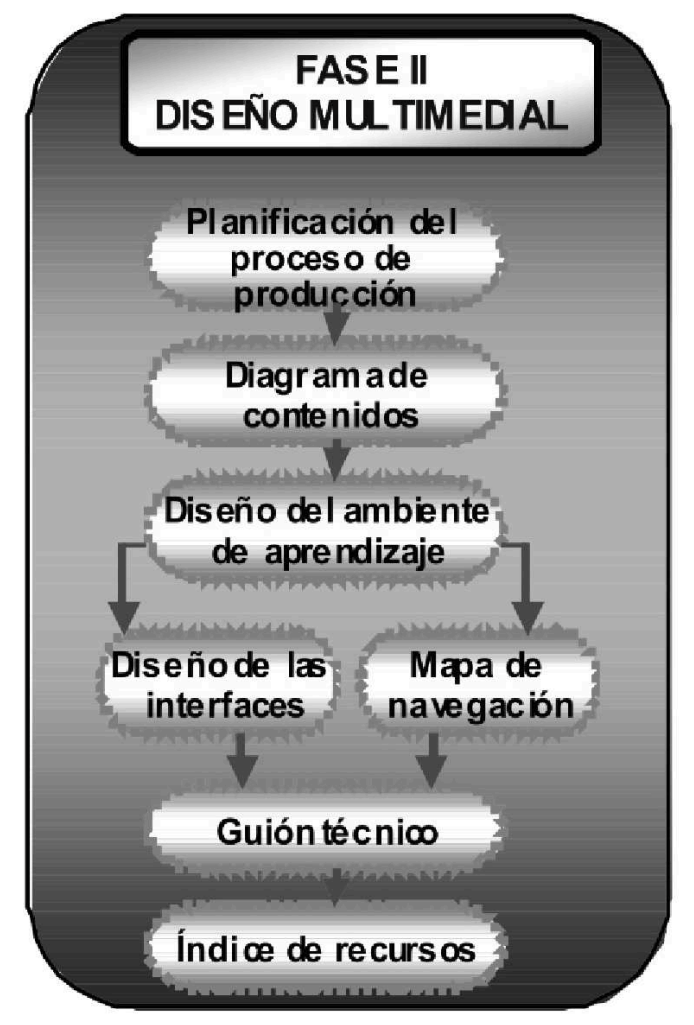

Figura 3. Fase II. Diseño multimedial. (Edupmedia-2008)

\section{Planeación del proceso de producción}

En todo proceso de desarrollo de software educativo se necesita planificar como quedará constituido $u$ organizado el desarrollo de las actividades, esto ayuda a que durante el proceso se tenga un control, que permita cumplir a cabalidad lo establecido en la planificación de la producción. El conjunto de procesos integra las metas u objetivos que se pretenden alcanzar, la distribución de las funciones que cada persona tendrá que asumir para lograr los objetivos que han sido planteados y por ultimo un cronograma de actividades.

\section{Diagrama de contenidos}

La funcionalidad de los diagramas es representar gráficamente la información contenida en un estudio temático de tal forma que se hace necesario complementar el diseño de un software con un esquema que represente los contenidos que se encuentran en la fase del diseño de contenidos. Esta representación se puede esquematizar de distintas formas ya sea por medio de mentefactos, mapas conceptuales y mapas mentales.

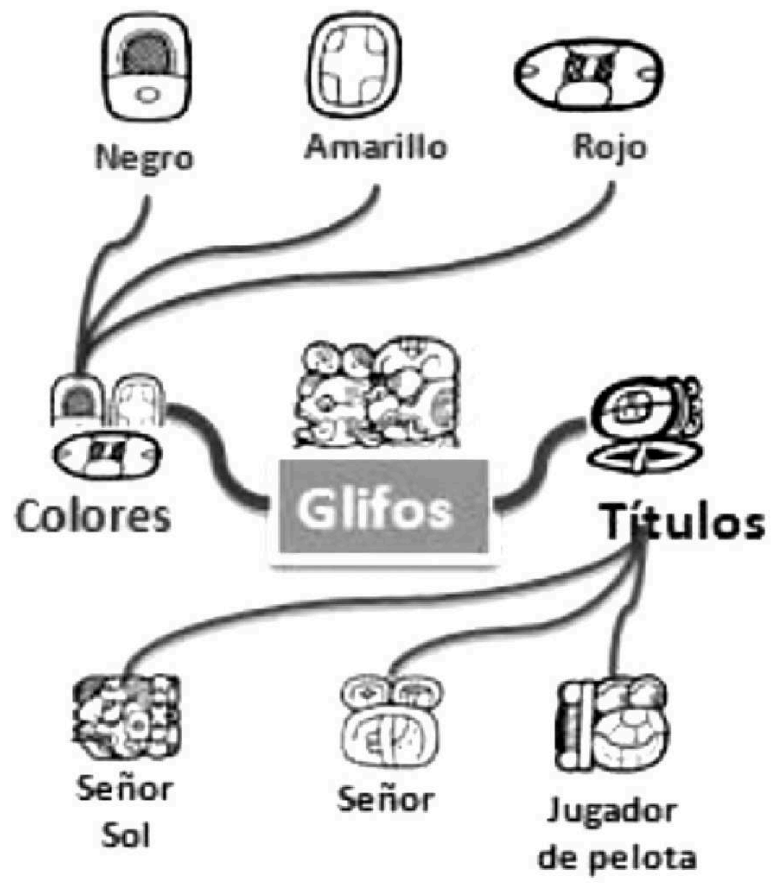

Figura 4. Ejemplo resumido de un mapa mental utilizado como diagrama de contenidos. (Software Lenguaje Maya Edupmedia-2008)

\section{Diseño del ambiente de aprendizaje}

Esta etapa comprende el conjunto de directrices que permitirán una comunicación directa entre el usuario y la computadora, ya que se presentará visiblemente el diseño de cada una de las interfaces gráficas de usuario. Estas directrices se identifican secuencialmente iniciando con el diseño de la ventana estándar para seguir con cada ventana o interfaz y para finalizar con el diseño del mapa de navegación

\section{Mapa de navegación}

El mapa de navegación de un software es una guía gráfica que se elabora para brindarle al usuario un fácil manejo del material computacional. Este esquema muestra la forma como están organizadas las interfaces gráficas de usuario, dentro de este esquema se incluye la imagen de la ventana principal y el despliegue de las demás cuando hay un hipervínculo que la interconecta.

\section{Guión técnico}

El guión técnico multimedial es un escrito que contiene detalladamente que es lo que queremos mostrar en la pantalla de nuestro computador. En el guión se escribe cómo va a ser cada "pantallazo", eso es lo que vamos a ver cada vez que interactuemos con el entorno. Debemos escribir todo, cómo van a ser los fondos, botones, sonidos, fotografías, 